\title{
The extracorporeal membrane oxygenation (ECMO) high-fidelity simulator: the best complementary tool to learn the technique
}

\author{
Santiago Montero ${ }^{1,2}$, Alain Combes ${ }^{2}$, Matthieu Schmidt ${ }^{2}$ \\ ${ }^{1}$ Acute and Intensive Cardiovascular Care Unit, Department of Cardiology, Hospital de la Santa Creu i Sant Pau, Biomedical Research Institute IIB- \\ Sant Pau, Universitat Autònoma de Barcelona, Barcelona, Spain; ${ }^{2}$ Medical Intensive Care Unit, iCAN, Institute of Cardiometabolism and Nutrition, \\ Hôpital de la Pitié-Salpêtrière, Assistance Publique-Hôpitaux de Paris, Université Pierre-et-Marie-Curie, Paris, France \\ Correspondence to: Matthieu Schmidt, MD, PhD. Service de Réanimation Médicale, iCAN, Institute of Cardiometabolism and Nutrition, Hôpital de \\ la Pitié-Salpêtrière, 47, bd de l'Hôpital, 75651 Paris Cedex 13, France. Email: matthieu.schmidt@aphp.fr. \\ Provenance: This is an invited Editorial commissioned by Section Editor Dr. Lei Huang (Cardiac Center of Tianjin Third-Central Hospital, \\ Tianjin, China). \\ Comment on: Zakhary BM, Kam LM, Kaufman BS, et al. The Utility of High-Fidelity Simulation for Training Critical Care Fellows in the \\ Management of Extracorporeal Membrane Oxygenation Emergencies: A Randomized Controlled Trial. Crit Care Med 2017;45:1367-73.
}

Submitted Oct 03, 2017. Accepted for publication Oct 16, 2017.

doi: $10.21037 /$ jtd.2017.10.117

View this article at: http://dx.doi.org/10.21037/jtd.2017.10.117

Extracorporeal membrane oxygenation (ECMO) use in intensive care units (ICUs) has expanded exponentially over the past decade (1) because of increasing scientific evidence $(2,3)$, technological advances and broadened indications (4-6). Its complex management and the need for rapid decision-making processes to counter complications require critical care stakeholders to be constantly updated and trained to care for patients in need of such interventions. Despite the highly challenging management of ECMO patients, well-established training programs are scarce $(7,8)$. To date, the Extracorporeal Life Support Organization, an international body focused on ECMO research and education, recommends traditional educational approaches with lectures and practice with water-drills or animal testing (9). However, because troubleshooting simulations and animal testing are usually expensive and available less often, water-drill-based ECMO training might be limited. On the other hand, because it seems to be highly effective, with long-lasting take-away skills, ECMO-simulation-based apprenticeship could be particularly useful for intensivists $(10,11)$. Using this pedagogical tool to train ECMO users could offer promising outcomes but have rarely been studied $(12,13)$. In addition, most published studies lacked control subjects to scientifically compare to simulation-training performance in the context of ECMO $(12,13)$.
To address those limitations, Zakhary et al. (14) recently published the first prospective randomized-controlled trial on simulation-based ECMO training. They hypothesized that a high-fidelity simulation-based ECMO apprenticeship would be superior to traditional water-drills. Twenty-one young critical care fellows without prior ECMO experience were enrolled in their study. The water-drill setup was an isolated ECMO circuit (i.e., a closed-loop circuit with pumped fluid). In contrast, the simulation circuit was connected to a highly realistic manikin ( $\operatorname{SimMan} 3 \mathrm{G}$ ), intubated and connected to a ventilator, with vital signs, respiratory and circuit data displayed on a monitor. Participants from both groups first took a written test to evaluate their baseline knowledge, followed by a scored simulated ECMO-emergency scenario (Sim1 recirculation). They then benefited from three ECMO lectures (ECMO basics, circuit and complications) before being randomized to the simulation or traditional training group. Thereafter, both groups were exposed to three ECMO-emergency scenarios with real-time circuit variables, with the simulation group also receiving additional real-time vital signs and ventilator settings.

Six weeks and 1 year later, the full initial written exam was repeated, followed by two scored ECMO scenarios (Sim2, the previously encountered pump failure; and Sim3, a novel pump-insufficiency scenario). Assessments 
of cognitive and technical skills to manage emergencies were scored blindly. Lastly, all participants rated their educational experience using a 9-item, 5-point, Likert-scale questionnaire. The primary outcome was the time required to perform critical actions, with secondary outcomes being scores of written knowledge tests, simulated scenarios and participants' questionnaires.

Few participants correctly performed the required actions before ECMO lectures and Sim1 practice ECMO scenario. Six weeks after randomization, all participants managed to adequately switch the backup pump (Sim2), with the simulation group performing faster [127 s (117-147 s) vs. $174 \mathrm{~s}(146-250 \mathrm{~s}) ; \mathrm{P}=0.004]$; that difference persisted at 1 year. When students were confronted with an unknown ECMO issue (i.e., Sim3 pump insufficiency), the appropriate critical action (fluid bolus) was significantly more frequently instigated by simulation-group participants at 6 months and 1 year, respectively. Similar scores evaluating emergency scenarios had been reported for the two groups before randomization. Pertinently, the simulation group achieved better results at 1 year for Sim2 [50\% (25-67\%) vs. 33\% (17-33\%); $\mathrm{P}=0.03]$ and $\operatorname{Sim} 3$ scenarios [58\% (42-75\%) vs. $25 \%(17-46 \%) ; \mathrm{P}=0.02]$. Moreover, simulation-group participants' questionnaire responses much more favorably rated the educational experience than those of the traditional group, especially for scenario realism and applicability.

Based on those results, the authors concluded that both ECMO-training methods were beneficial but with the high-fidelity simulation-based apprenticeship performing better. Those observations warrant several comments. First, when the same scenario was repeated 6 weeks and 1 year later, no scoring differences were found, emphasizing the positive effects of both ECMO-learning methods. However, important differences were observed when the students faced an unknown emergency scenario (i.e., pump insufficiency). Surprisingly, this common complication was detected and solved by only $1(9 \%)$ of the 11 traditional group trainees. Such a low percentage of appropriate reactions raises the question of the usefulness of a conventional water-drill apprenticeship, because cannula tilting, reflecting hypovolemia or malposition, is a very frequent ECMO event. Second, although the simulation group obtained better written-test grades at 1 year, the primary outcome was evaluated on a manikin, which might have contributed to their better outcomes because they were already familiar with this exercise. The authors justified this choice to best mimic a real-life situation. Finally, the last notable drawback of this study remains the small number of participants in both groups. Further studies including more participants are needed to confirm the beneficial use of ECMO simulation for training.

In addition to reporting a benefit of the high-fidelity simulation-based ECMO apprenticeship, Zakhary et al. highlighted relevant concepts of medical education. In the late 1980s, the concept of "situation awareness" was defined as "the ability of an individual to maintain an adequate internal representation of the status of the environment in complex, dynamic circumstances" (15). Briefly, situation awareness has three hierarchical levels including perception (Level 1), comprehension (Level 2) and projection (Level 3). In the ICU, suitable situation awareness is crucial to avoid preventable errors. The authors of a recent study (16) found simulation-based training to be superior to lecture-based when teaching situation awareness, primarily because of improved perception ability. The better perception performance and global situation awareness might explain the faster response of the simulation-based group described by Zakhary et al. According to a recent review of critical care medicine incidents, perception-related situation-awareness errors were slightly more frequent (17). Although it has not been fully demonstrated, decision-making processes should be improved when perception is better.

In the context of ECMO, low patient volume and the high mortality associated with its complications make recognition of the latter challenging. The ability of ICU physicians to adequately react to those dreadful situations is determined by previous exposure, which contributes to rapid recognition and identification of the scenarios. All ICU stakeholders, including experienced and novice apprentices, have reported enthusiastic endorsement of simulation in the critical care environment $(14,16,18,19)$. Because traditional teaching strategies overemphasize cognitive skills, while ignoring behavioral ones (19), simulation could combine both. This approach is particularly relevant in a context requiring rapid and adequate decision-making processes, like ECMO. Hence, a participative, immersive, interactive and realistic environment may foster the development of long-lasting technical skills, as shown in the paper of interest. However, the benefit on time to critical action remains unclear, since Anderson et al. (19) reported no impact on time responses of experienced specialists after simulated-based ECMO training.

In this study, Zakhary et al. elegantly demonstrated the benefit of a practical, hands-on, immersive experience 
in strengthening behavioral ECMO skills. A visual, engaging and realistic environment offers adults the sense of realism, crucial keys to enhancing learning experiences and maintaining long-term performance. However, their promising results should not rule out the major benefit of high-volume, bedside teaching, as nothing can be more realistic than "real" life. Hence, high-quality simulationbased ECMO apprenticeships should be seen as the best complementary training method for both novice and experienced physicians. The extent of its use for the nursing staff involved in ECMO care now deserves further studies.

\section{Acknowledgements}

S Montero is funded by a 2016 Clinical Training Grant awarded by the European Society of Cardiology.

\section{Footnote}

Conflicts of Interest: Dr. Combes is the primary investigator of the randomized EOLIA trial (NCT01470703) on VVECMO partially supported by Maquet. Drs. Combes and Schmidt have received honoraria for lectures from Maquet. Dr. Montero has no conflicts of interest to declare.

\section{References}

1. MacLaren G, Combes A, Bartlett RH. Contemporary extracorporeal membrane oxygenation for adult respiratory failure: life support in the new era. Intensive Care Med 2012;38:210-20.

2. Peek GJ, Mugford M, Tiruvoipati R, et al. Efficacy and economic assessment of conventional ventilatory support versus extracorporeal membrane oxygenation for severe adult respiratory failure (CESAR): a multicentre randomised controlled trial. Lancet 2009;374:1351-63.

3. Pham T, Combes A, Roze H, et al. Extracorporeal membrane oxygenation for pandemic influenza $\mathrm{A}(\mathrm{H} 1 \mathrm{~N} 1)$ induced acute respiratory distress syndrome: a cohort study and propensity-matched analysis. Am J Respir Crit Care Med 2013;187:276-85.

4. Fuehner T, Kuehn C, Hadem J, et al. Extracorporeal membrane oxygenation in awake patients as bridge to lung transplantation. Am J Respir Crit Care Med 2012;185:763-8.

5. Lang G, Ghanim B, Hotzenecker K, et al. Extracorporeal membrane oxygenation support for complex tracheo- bronchial proceduresdagger. Eur J Cardiothorac Surg 2015;47:250-5; discussion 256.

6. Rinieri P, Peillon C, Bessou JP, et al. National review of use of extracorporeal membrane oxygenation as respiratory support in thoracic surgery excluding lung transplantation. Eur J Cardiothorac Surg 2015;47:87-94.

7. Muratore S, Beilman G, John R, et al. Extracorporeal membrane oxygenation credentialing: where do we stand? Am J Surg 2015;210:655-60.e2.

8. Weems MF, Friedlich PS, Nelson LP, et al. The Role of Extracorporeal Membrane Oxygenation Simulation Training at Extracorporeal Life Support Organization Centers in the United States. Simul Healthc 2017;12:233-9.

9. ELSO Guidelines for Training and Continuing Education of ECMO. 2010.

10. Fehr JJ, Boulet JR, Waldrop WB, et al. Simulation-based assessment of pediatric anesthesia skills. Anesthesiology 2011;115:1308-15.

11. Weinger MB, Banerjee A, Burden AR, et al. Simulationbased Assessment of the Management of Critical Events by Board-certified Anesthesiologists. Anesthesiology 2017;127:475-89.

12. Burkhart HM, Riley JB, Lynch JJ, et al. Simulation-based postcardiotomy extracorporeal membrane oxygenation crisis training for thoracic surgery residents. Ann Thorac Surg 2013;95:901-6.

13. Burton KS, Pendergrass TL, Byczkowski TL, et al. Impact of simulation-based extracorporeal membrane oxygenation training in the simulation laboratory and clinical environment. Simul Healthc 2011;6:284-91.

14. Zakhary BM, Kam LM, Kaufman BS, et al. The Utility of High-Fidelity Simulation for Training Critical Care Fellows in the Management of Extracorporeal Membrane Oxygenation Emergencies: A Randomized Controlled Trial. Crit Care Med 2017;45:1367-73.

15. Endsley MR, editor. Design and evaluation for situation awareness enhancement. Proceedings of the Human Factors Society annual meeting; 1988. SAGE Publications Sage CA: Los Angeles, CA.

16. Lee Chang A, Dym AA, Venegas-Borsellino C, et al. Comparison between Simulation-based Training and Lecture-based Education in Teaching Situation Awareness. A Randomized Controlled Study. Ann Am Thorac Soc 2017;14:529-35.

17. Schulz CM, Krautheim V, Hackemann A, et al. Situation awareness errors in anesthesia and critical care in 200 cases of a critical incident reporting system. BMC Anesthesiol 2016;16:4. 
18. Fehr JJ, Shepard M, McBride ME, et al. Simulation-Based Assessment of ECMO Clinical Specialists. Simul Healthc 2016;11:194-9.

19. Anderson JM, Boyle KB, Murphy AA, et al. Simulating

Cite this article as: Montero S, Combes A, Schmidt M. The extracorporeal membrane oxygenation (ECMO) highfidelity simulator: the best complementary tool to learn the technique. J Thorac Dis 2017;9(11):4273-4276. doi: 10.21037/ jtd.2017.10.117 extracorporeal membrane oxygenation emergencies to improve human performance. Part I: methodologic and technologic innovations. Simul Healthc 2006;1:220-7. 